\title{
Expert Discussion: SABCS 2021
}

\author{
Rupert Bartsch ${ }^{a}$ Cornelia Kolberg-Liedtke ${ }^{b, c}$ Guy Jerusalem ${ }^{d} \quad$ Carlo Palmieri $^{e, f}$ \\ Wolfgang Jannig \\ ${ }^{a}$ Division of Oncology, Department of Medicine I, Medical University of Vienna, Vienna, Austria; ${ }^{\mathrm{b}}$ Department \\ of Gynecology and Obstetrics, University Hospital Essen, Essen, Germany; 'Phaon Scientific GmbH, Wiesbaden, \\ Germany; ${ }^{d}$ Division of Medical Oncology, Department of Medicine, CHU Sart Tilman Liège and Liège University, \\ Liège, Belgium; ${ }^{e}$ Department of Molecular and Clinical Cancer Medicine, Institute of Systems, Molecular and \\ Integrative Biology, University of Liverpool, Liverpool, UK; ${ }^{\mathrm{f}}$ The Clatterbridge Cancer Centre NHS Foundation Trust, \\ Liverpool, UK; ${ }^{9}$ Department of Obstetrics and Gynecology, University Hospital Ulm, Ulm, Germany
}

\section{Bartsch: What Was Your Personal Highlight from the 2021 SABCS?}

Kolberg-Liedtke: My personal highlight was the presentation of Gianpaolo Bianchini, who presented results of his translational analysis of the NeoTRI-PaPDL1 study (NCT02620280), which aimed to generate predictive information regarding response to neoadjuvant atezolizum$\mathrm{ab}$ therapy using imaging mass cytometry (IMC). IMC allows the study of protein expression at the single-cell level and the location of the cells within the tumor microenvironment (TME). One must acknowledge that clinical use of this technology is yet limited, and interpretation has to be done with caution; however, results demonstrate feasibility of this technology in the context of a clinical trial. Personally, I am particularly delighted to see those results given that translational analyses of our own clinical atezolizumab trial neoMono will also use IMC technology for therapy response prediction. neoMono is an active open randomized phase 2 study that will recruit up to 458 (male or female) patients with early, therapy-naïve, triple-negative breast cancer or breast cancer with a hormone receptor positivity of less than $10 \%$ with the goal to analyze the association between addition of a monotherapy window with atezolizumab prior to a combination of chemotherapy (anthracycline, taxane, and platinum) and atezolizumab with pathologic complete remission as primary study endpoint (defined as lack of invasive tumor cells and lymph node). Throughout the trial, longitudinal parallel liquid and tissue biopsies will provide a basis for identification/validation of predictive biomarkers.
Jerusalem: Although we are somewhat disappointed about the outcome, we have seen the first phase 3 trial data reported concerning a SERD compared to standard endocrine therapy. Elacestrant, an oral selective estrogen receptor degrader was compared in this study to investigator's choice of endocrine monotherapy for ER+/HER2advanced/metastatic breast cancer following progression on prior endocrine and CDK4/6 inhibitor therapy. A statistically significant improvement in median progression-free survival from 1.91 to 2.79 months was observed. In my opinion, this trial has shown that any endocrine monotherapy is not an appropriate treatment option for an unselected patient population after a CDK4/6 inhibitor-based treatment for advanced/metastatic disease. However, some patients remain endocrine sensitive, and for them elacestrant gives a higher chance of longer-term disease control. We need to better understand how to select the patients who benefit most from this SERD and which are the characteristics of patients who develop an ESR1 mutation. Based on the results of this trial (EMERALD), I look forward to trials evaluating therapy by SERD in the adjuvant setting.

Palmieri: For me, the highlight was the data from studies which utilized cell-free DNA to change treatment or look at outcome/relapse. The French PADA-01 study which randomized patients to either continuation of endocrine treatment with palbociclib plus an aromatase inhibitor versus switch to fulvestrant plus palbociclib based on the development of ESR1 mutations in ctDNA demonstrated that progression-free survival was superior in patients who were switched to fulvestrant,11.9 months, as 
compared to 5.7 months in patients without the switch (stratified HR 0.68, $p=0.005$ ). This for me demonstrates that utilizing "molecular relapse" rather than "radiological relapse" to define treatment change could lead to better clinical outcomes for our patients. While the Italian BioItaLEE trial demonstrated the possible value of monitoring the dynamic of ctDNA in patients with metastatic breast cancer. Within the BioItaLEE trial, patients with hormone receptor-positive/HER2-negative metastatic breast cancer and treated first line with ribociclib plus letrozole had cfDNA measured over time. In essence, the study demonstrated that the presence of a detectable mutation at baseline was a negative prognostic factor, while patients who had no detectable mutations at day 15 had a lower risk of risk of progression (HR: 0.53; 95\% CI 033 $0.86)$. For me, these data demonstrate that we may be able to move to a situation where we can triage patients based on an early molecular analysis of the cfDNA and so maybe allow us to have a more nuanced approach to the follow-up of ER-positive/HER2-negative patients with metastatic breast cancer in the first-line setting. An interesting question is can we forego regular radiological follow-up in such patients, while on the other hand we can identify patients who may need to have an early change of treatment?

PADA-01 and BioItaLEE trial show us that we are moving to an era where progression or lack of therapy benefit will be defined based on cfDNA rather than radiology. Such a move to molecular rather than radiologically defined relapse will lead to a number of challenges both in our day-to-day clinically practice and research, but it will also help us better use our finite healthcare resources more efficiently. While in the early disease setting, cTRAK demonstrates we can use cfDNA detection to predict relapse in triple-negative breast cancer, this will afford exciting opportunities to try to intervene and prevent relapse, although earlier testing with more sensitive assays is likely to be needed.

Janni: My personal highlight was the French PADA-01 study. In this study, 172 patients with rising ESR1 mutations in ctDNA in peripheral blood were randomized between the continuation of endocrine treatment with palbociclib plus an aromatase inhibitor versus switch to fulvestrant plus palbociclib. The progression-free survival in patients who were switched to fulvestrant was 11.9 months, compared to 5.7 months in patients without the switch (stratified HR 0.68, $p=0.005$ ).

This study, in my view is an important and successful proof of principle study that we might be able to individualize treatment of metastatic breast cancer in future, based on ctDNA in peripheral blood. The global SERENA6 study will investigate this hypothesis further with an oral SERD.

\section{Bartsch: What Is the Clinical Impact of the Metanalyses of Aromatase Inhibitors versus Tamoxifen as Adjuvant Endocrine Therapy in Premenopausal Patients and the Role of Anthracyclines as a Component of Adjuvant Chemotherapy?}

Kolberg-Liedtke: In a meta-analysis based on data of 7,030 premenopausal women included in the ABCSG 12, TEXT, SOFT, and HOBOE trials at a median follow-up of 8 years, treatment with an AI was associated with a significant $21 \%$ reduction in the risk for recurrence (defined as distant, locoregional, or new contralateral breast cancer) compared with tamoxifen. However, the authors observed no significant difference in breast cancer-specific mortality risk, with comparable 10 -year rates of 6.8 and $7.2 \%$ in the $\mathrm{AI}$ and tamoxifen groups, respectively. These data are in line with most data among premenopausal (provided that ovarian suppression is performed) and postmenopausal data in that aromatase inhibitors are superior to tamoxifen in most, but not all survival endpoints (particularly overall survival). Personally, I have followed a risk-based approach to decide whether AI or tamoxifen is applied in premenopausal women and will continue to do so after SABCS in that a higher risk of recurrence will justify indication of aromatase inhibitors. However, patients should be well-informed regarding risks/side-effects and should be included in the decision-making process.

Jerusalem: Concerning aromatase inhibitors, it becomes more and more evident that they are associated with improved long-term outcome compared to tamoxifen (reduces the relative risk of breast cancer recurrence by about $21 \%$ ) and relative reduction in distant recurrence by $17 \%$, but no effect on breast cancer mortality or overall survival has currently been observed. On the other hand, they are also associated with more bone fractures. Longer follow-up is needed to see the effect on overall survival. In my experience, it is not easy to treat young premenopausal patients with ovarian function suppression and aromatase inhibitor therapy, but if the patient is able to support the treatment, this should be the preferred one for high-risk patients. Evaluation of compliance and optimization of side effect management is important in order to avoid that patients stop definitively any endocrine therapy. Even if the benefit is a little bit less pronounced with alternative endocrine therapies, the benefit is still very high compared to no further adjuvant endocrine therapy.

Concerning the meta-analysis of anthracycline-based regimens compared to anthracycline-free regimens, I'm really surprised and somewhat confused about the outcome. According to the results, only concomitant but not sequential use of anthracyclines and taxanes is associated with improved outcome compared to anthracycline-free 
regimens. Nevertheless, in our institution, we definitively stopped any concomitant administration because the toxicity is very high. A limitation of the meta-analysis is that the safety profile is not included in the analysis. In Europe, anthracyclines are still very popular. I'm not ready to either delete or use only concomitant anthracycline/taxane-based regimens. However, more than ever, we should use anthracyclines only with caution in patients at higher risk of developing cardiac problems, or probably we should preferentially use anthracycline-free regimens in these patients. Unfortunately, we are still lacking predictive biomarkers for the specific benefit related to anthracycline-based therapy.

Palmieri: The Oxford overview groups meta-analysis reported that in premenopausal women with early breast cancer, OS plus an AI was superior to tamoxifen in terms of disease-free survival and distant disease-free survival, but no differences were seen in breast cancer-specific mortality or overall mortality. The group presented data on bone fracture, but no other patient-level data on toxicity and quality of life was available.

At SABCS 2021, updated analysis of the TEXT/SOFT trials was also presented, and in an analysis based on HER2 status and in the HER2-positive population, 12year overall survival appeared to favor OS plus tamoxifen as compared to OS plus AI. While in the HER2-negative group who received chemotherapy, an absolute $+3.3 \%$ improvement in overall survival in favor of OS plus exemestane was observed. Of note, similar data in the HER2-positive breast cancers was reported in HOBOE. No data was presented based on HER2 status by the Oxford overview group.

Based on all these data in premenopausal women with HER2-positive disease, utilizing OS plus tamoxifen would seem a reasonable option. While in those with HER2negative disease who have received chemotherapy, I would aim to use OS plus AI. However, the key issue with regard to endocrine therapy in young women is giving a treatment that they can tolerate and comply with consistently, therefore starting patients on OS plus tamoxifen and then switching to an AI to assess tolerability. If there are tolerability issues despite optimal management, then I would switch back to tamoxifen. We need to bear in mind that in some women it may be necessary to discontinue ovarian suppression if there are significant toxicity issues. Moving forward, and something we need to pay much more attention to is ensuring optimal management of endocrine therapy-related toxicity in young women on OS. This is going to be key to ensure compliance and maximize the benefit of this treatment. Ongoing research in this area is something we also need to see more in the future.

A meta-analysis demonstrated a significant benefit of taxanes plus anthracycline versus a taxane without an- thracycline with regard to recurrence and breast cancer mortality. However, when concurrent versus sequential anthracyclines regimens were compared, benefit was only seen for concurrent regimens. However, it should be noted that the cumulative dose of docetaxel within the sequential anthracycline/taxane regimens was lower, 300 $\mathrm{mg} / \mathrm{m}^{2}$, as compared to the dose within the concurrent regimen, docetaxel plus cyclophosphamide, where it was $4,500 \mathrm{mg} / \mathrm{m}^{2}$. Furthermore, in the concurrent schedules, the cumulative doses of anthracycline and taxane were higher than in the sequential schedules. Therefore, cumulative dose seems to be important. Furthermore, the sequential taxanes plus anthracycline regimes were predominately 3 weekly schedules rather than dose dense. Previous data from the Oxford Overview group has demonstrated that sequential regimens if they were dosedense regimens were similar if not a bit better than concurrent regimens. A key limitation of these data was the lack of individual level data on safety or quality of life. The known toxicities associated with anthracycline and taxane combination regimens would limit the widespread use of such regimens. Taking all the available data together, we should be aiming to utilize dose-dense regimens in sequential anthracyclines-taxanes regimens. It would be preferable to try using the anthracycline and taxane combination.

Janni: The meta-analysis on aromatase inhibitors versus tamoxifen in combination with OFS as adjuvant endocrine therapy in premenopausal patients demonstrated a significant benefit in disease-free survival (10-year absolute difference 14.7 vs. 17.5\%) and distant diseasefree survival (10-year absolute difference 10.2 vs. $12.1 \%$ ) but did not demonstrate any difference in the breast cancer-specific mortality. In the light of increased morbidity with aromatase inhibitors and in the context of the reported data of the SOFT/TEXT trials, I think that most patients are optimally treated with tamoxifen and OFS. However, the option of aromatase inhibitors and OFS should be discussed on an individual basis.

The meta-analysis on the role of anthracyclines demonstrated a significant and relevant benefit of anthracyclines in concurrent regimes, both for disease-free survival (10-year absolute difference 12.3 vs. $21.0 \%$ ) and breast cancer-specific mortality (10-year absolute difference 9.9 vs. $13.2 \%$ ). However, no benefit was seen for sequential regimen with reduced cumulative taxane dosage. Therefore, we may assume that regimens, such as TAC have a higher efficacy, but regimens, such as ECPac and DC are equally effective. A major limitation of the analysis is the lack of solid toxicity data and the inclusion of dose-dense regimen. In conclusion, I am reluctant to use TAC because of the toxicity in most patients. 


\section{Bartsch: In the Light of Recent Data, How Can We Optimize the Use of Immune Checkpoint Inhibitors in Breast Cancer?}

Kolberg-Liedtke: As with the use of aromatase inhibitors versus tamoxifen, clinical practice with checkpoint inhibition should not be adjusted after SABCS 2021. We must acknowledge that the analysis of Gianpaolo Bianchini and others suggests that predictive biomarkers beyond PD-L1 expression may be identified, and results of Keynote-522 regarding an improved iDFS with adjuvant use of pembrolizumab provide ONE signal that adjuvant ICI therapy may further improve efficacy of neoadjuvant ICI therapy; however, additional subgroup analyses as well as data from other adjuvant ICI trials need to support these observations.

Jerusalem: It is first a great achievement that immunotherapy is now also standard of care for triple-negative breast cancer where we have many unmet needs. I see many open questions. First, for patients who received neoadjuvant chemo/immunotherapy, do we really need to continue checkpoint inhibitors after surgery in patients who present a complete pathological response? Another question is if we should add a checkpoint inhibitor to chemotherapy in the adjuvant setting for patients who received only neoadjuvant chemotherapy (smaller tumors not eligible for upfront checkpoint inhibitor therapy but without a pathological complete remission) or in the adjuvant setting for high-risk patients having had upfront surgery. Concerning more specifically the KEYNOTE-522 study evaluating neoadjuvant pembrolizumab + chemotherapy compared to placebo + chemotherapy, patients received only adjuvant pembrolizumab, but we know that capecitabine (in case of residual disease) or olaparib (for high-risk BRCA mutated patients) are also useful treatment options. Should we add these options to immunotherapy in selected patients? What is the expected benefit with this therapy escalation? Finally, we also wish to better understand which are the most effective chemotherapy regimens to be combined with checkpoint inhibitors.

Palmieri: For me, this is split into two parts: firstly the implementation of what we already know, and secondly how we further develop and refine the use of immune checkpoint inhibitors in breast cancer. With regard to key steps needed to put into practice what we already know, the first of these is ensuring the implementation of a quality-assured methodology for performing PDL1 immunohistochemistry utilizing the appropriate antibody and scoring system for the checkpoint inhibitor in question. This is absolutely key to ensuring that we identify all eligible patients. The second key challenge is ensuring education in the breast community on the appropriate management of IO toxicity both to ensure patient safety but also to ensure patients aren't inappropriately stopped. The latter is particularly important in the early disease setting. Given immune checkpoint inhibitors have been widely used in other tumor types, the vast majority of hospitals will have the appropriate protocols in place although some education. Finally, understanding if there are any long-term toxicities in early breast cancer will also be important.

With regard to the development and refinement of immune checkpoint inhibitors in the early disease setting, issues that need to be address include: (1) trying to understand if everyone needs an immune checkpoint inhibitor. In those who have a pCR following neoadjuvant therapy, can we stop checkpoint inhibitors following surgery? (2) What is the optimal chemotherapy backbone for these agents? (3) Understanding if there is a benefit or not of adjuvant capecitabine in combination with pembrolizumab (gBRCAwt) in these patients with residual disease after neoadjuvant treatment will be key, as will be the question of how best to combine immune checkpoint inhibitors with olaparib in patients with gBRCA mutation. Based on the results of cTRAK, we need to understand the benefit of pembrolizumab in those who become cfDNApositive during treatment.

Janni: In my view, the use of pembrolizumab in patients with early-stage triple-negative breast cancer in analogy to the inclusion criteria of the Keynote 522-study will become standard of care. However, we still have very little data from patients who receive post-neoadjuvant capecitabine and no data from patients with BRCA mutations and indication for olaparib. We urgently need data to support decision making in this space.

Following the study design presented by Nick Turner at this year's SABCS, we might even consider using immune checkpoint inhibitors in patients with molecular relapse based on ctDNA during surveillance.

\section{Bartsch: What Is Your Approach towards Systemic Therapy of HER2-Positive Breast Cancer Brain Metastases?}

Kolberg-Liedtke: Although the DESTINY-Breast-03 trial has demonstrated particular efficacy of trastuzumab-deruxtecan among patients with stable brain metastases, the HER2CLIMB clinical trial remains the only large clinical trial to date suggesting efficacy of a modern HER2-targeted therapy approach by use of tucatinib (in combination with trastuzumab and capecitabine) in patients with active breast metastases. This data is supported by results from TBCRC049 (NCT03501979) suggesting efficacy of tucatinib even in patients with leptomeningeal metastases. Therefore, as of now tucatinib represents the agent of choice for patients with active 
brain metastases, whereas in patients with stable brain metastases trastuzumab deruxtecan definitely is an option.

Jerusalem: First of all, it is useful to remember that fortunately we can offer several years of median overall survival to HER2-positive patients presenting brain metastases. We have also learned that all systemic therapies can have some antitumor effect once the patient presents macroscopic brain metastases. I'm of course excited about the impact of tucatinib when combined with trastuzumab and capecitabine on progression-free survival and overallsurvivalcompared to trastuzumaband capecitabine alone. We also have to congratulate the investigators that they included patients with active brain metastases, a subgroup of patients in general excluded from clinical trials. In the short term, this regimen is the second-line standard of care therapy for HER2-positive metastatic breast cancer with brain metastases. In the longer term, we have to wait for the ongoing trials evaluating trastuzumab deruxtecan in this patient subpopulation. Given the spectacular results in heavily pretreated patients and also signals of efficacy in patients with brain metastases, this agent has the potential to challenge a tucatinib-based regimen also in patients with active brain metastases, but of course we first have to wait for the outcome of the ongoing clinical trials.

Palmieri: The evolving evidence base regarding the activity of systemic treatments for CNS disease in HER2positive breast cancer is important and good news for our patients who develop CNS disease, but clearly there is still much more to be done.

HER2CLIMB has clearly demonstrated the intracranial activity of tucatinib when combined with trastuzumab and capecitabine in patients with stable as well as progressing CNS disease. However, its license limits its use to those patients who have received at least 2 prior anti-HER2 treatment regimens. Therefore, in those patients who present with CNS disease in the de novo setting or on first-line treatment and develop progressive intracranial disease after local therapy, the treatment in many healthcare systems will still be an ADC. The evolving CNS data from DESTINY-Breast 03 demonstrates that with regard to ADCs, trastuzumab deruxtecan is superior to T-DM1 in patients with CNS disease and so would be the preferred second-line treatment in such patients (in keeping with those for extracranial disease). However, we should note that the population with brain metastasis entered into DESTINYBreast 03 differed from those entered into HER2CLIMB with only clinically stable and treated allowed to enter DESTINY-Breast 03 in contrast to HER2CLIMB which included those with progressing or untreated CNS disease. Therefore, based on the current data as well as the license in Europe, an ADC in the form of T-DM1 (T-
Dxd when licensed in Europe) followed by tucatinib would be the current sequence approach I would take for HER2-positive CNS disease that has progressed after local therapy.

Janni: The data which were presented at this year's SABCS on the efficacy of trastuzumab deruxtecan in patients with brain metastases definitely looked very appealing to me, with the limitation that this was an exploratory analysis. On the other hand, the data of the HER2CLIMB study using the tucatinib combination also show a very favorable efficacy of this combination. My take: in patients without brain metastases, trastuzumab deruxtecan is the new standard of care in second line. In patients with brain metastases, I would currently favor the tucatinib combination if the toxicity profiles do not suggest using trastuzumab deruxtecan.

\section{Conflict of Interest Statement}

Rupert Bartsch

Advisory Role: Astra-Zeneca, Daiichi, Eisai, Eli-Lilly, MSD, Novartis, Pfizer, Pierre-Fabre, Puma, Roche, Seagen.

Lecture Honoraria: Astra-Zeneca, Eli-Lilly, Novartis, Pfizer, Pierre-Fabre, Roche, Seagen.

Research Support: Daiichi, MSD, Novartis, Roche.

\section{Cornelia Kolberg-Liedtke}

Honoraria: Roche, Genomic Health, GlaxoSmithKline, AstraZeneca, Eisai, Celgene, Amgen, Gedeon Richter, Novartis, Pierre Fabre, Teva.

Consulting or Advisory Role: Roche, Genomic Health, Teva, Pierre Fabre, Novartis. heim.

Research Funding: Eisai, Roche, Novartis, Boehringer Ingel-

Travel, Accommodations, Expenses: Roche, Teva, PharmaMar, Celgene.

Employment: Palleos healthcare (VP Medical and Scientific Development), Phaon scientific (Co-founder, Managing Director).

\section{Guy Jerusalem}

Research grants and/or honoraria from Novartis, Amgen, Roche, Pfizer, Bristol-Myers Squibb, Lilly, Astra-Zeneca, Daiichi Sankyo, Abbvie, Seagen.

\section{Carlo Palmieri}

Grant funding from Pfizer and Daiichi Sankyo and honoraria for advisory boards from Pfizer, Roche, Daiichi Sankyo, Novartis, AZ, Exact sciences, and Eli Lilly.

\section{Wolfgang Janni}

Research Grants and/or honoraria from Sanofi-Aventis, Novartis, Roche, Pfizer, Lilly, AstraZeneca, Chugai, GSK, Eisai, Cellgene, DaiichiSankyo, MSD, Seagen, Johnson\&Johnson. 


\section{Chair}

Prof. Rupert Bartsch

Clinical Research Unit

Division of Oncology

Department of Medicine I

Medical University of Vienna

Währinger Gürtel 18-20

AT-1090 Vienna, Austria

rupert.bartsch@meduniwien.ac.at

\section{Participants}

Prof. Cornelia Kolberg-Liedtke

Klinik für Frauenheilkunde und Geburtshilfe

Universitätsklinikum Essen (AöR)

Hufelandstrasse 55

DE-45147 Essen, Germany

cornelia1979@googlemail.com
Guy Jerusalem, $M D, P h D$

CHU Liège and Liège University

Domaine Universitaire du Sart Tilman, B35

BE-4000 Liège, Belgium

g.jerusalem@chuliege.be

Carlo Palmieri, $M D$

Department of Molecular and Clinical Cancer Medicine Institute of Systems

Molecular and Integrative Biology

University of Liverpool

Ashton Street

Liverpool L69 3GE, UK

C.Palmieri@liverpool.ac.uk

Prof. Wolfgang Janni

Department of Obstetrics and Gynecology

Universitätsklinikum Ulm

Prittwitzstrasse 43

DE-89075 Ulm, Germany

Wolfgang.Janni@uniklinik-ulm.de 